\title{
On Resultant Tones
}

This content has been downloaded from IOPscience. Please scroll down to see the full text. 1895 Proc. Phys. Soc. London 1493

(http://iopscience.iop.org/1478-7814/14/1/308)

View the table of contents for this issue, or go to the journal homepage for more

Download details:

IP Address: 128.178.131.113

This content was downloaded on 02/10/2015 at 08:26

Please note that terms and conditions apply. 
P.S.-No reference to Dr. Schmidt's original paper was given in the short notice published in the Report of the British Association. I had therefore supposed that the latter was a preliminary note. Professor Schuster has, however, recently shown to me Dr. Schmidt's complete investigation, and he has kindly calculated the current-density at latitudes $50^{\circ}$ and $55^{\circ}$ on the prime meridian from formulæ given by $\mathrm{Dr}$. Schmidt. The result is upward currents and 0.20 to 0.15 ampere per square kilometre at lat. $50^{\circ}$ and $55^{\circ}$ respectively. The mean of these two numbers, viz. $0 \cdot 175$, is nearly equal to Schmidt's mean for the whole earth $(0 \cdot 17)$. It is opposite in direction to and very much greater in magnitude than any vertical current the existence of which is compatible with the results of the Magnetic Survey.

\section{Discussion.}

Mr. Watson said a few words on the difficulty experienced in determining the line-integral in South Wales, due to the presence of closed curves.

\section{On Resultant Tones.}

By Professor J. D. Everett, F.R.S.*

1. The received theory of the generation of resultant tones in the ear may be summed up with rough accuracy as followst:-

The drumskin being pulled inwards by the end of the bandle of the "hammer," which is attached to its centre, offers unsymmetrical resistance to displacement in the inward and outward directions, so that the equation for the movement of its centre in free vibration would be

or to a closer approximation

$$
-\ddot{x}=\omega^{2} x+\alpha x^{2},
$$

$\omega, \alpha, \beta$ being constants.

$$
-\ddot{x}=\omega^{2} x+\alpha x^{2}+\beta x^{3},
$$

* Read January 24, 1896.

† See Tonenppfindungen, Appendix xii. ; Rayleigh on Sound, art. 68; Bosanquet, Proc. Phys. Soc. vol.iv. p. 240, arts. 57-69. 
The value of $\omega$ when the second is the unit of time is less than 60 , hence the frequency of free vibration, being $\omega / 2 \pi$, is less than 10.

When two barmonic forces of frequencies $m$ and $n$ act upon the drumskin, they produce, in addition to their own tones, certain "resultant tones," the one of largest amplitude being the "first difference-tone," of frequency $n-m$. The next largest amplitude is about $\left(\frac{n-m}{n+m}\right)^{2}$ of this, and belongs to the "first summation-tone," of frequency $n+m$. Neither of these tones will be audible unless the excursion $x$ is so large that $\alpha x^{2}$ is sensible in comparison with $\omega^{2} x$. There will also be difference-tones of frequencies $2 m-n$ and $2 n-m$, but neither of these will be audible unless $\beta x^{3}$ is sensible compared with $\omega^{2} i v$.

2. This theory does not appear sufficient to account for the loud resultant tones which are sometimes heard. When a Helmboltz siren is driven rapidly, with the rows of holes 9 , 12,15 , and 18 open, the resultant tone of frequency 3 on the same scale is the most prominent tone in the whole volume of sound.

3. The view which I desire to put forward is closely connected with the well-known theorem of Fourier, that every periodic variation can be resolved in one definite way into harmonic constituents, whose periods must be iucluded in the list $1, \frac{1}{2}, \frac{1}{3}, \frac{1}{4}, \&$ c., where 1 denotes the period of the given variation itself. The corresponding frequencies will be as $1,2,3,4$, \&c.

In the majority of cases, when this analysis is carried out, the fundamental constituent, represented by 1 in the above lists, is the largest or among the largest; but in the case of a variation compounded of two simple tones with frequencies in the ratio of two integers, neither being a multiple of the other, the fundamental will be absent, and the Fourier series will consist of only two terms, which in the language of acoustics are harmonics of the fundumental.

4. Clearness of thought is facilitated in these matters by supposing a curve to be drawn, in which horizontal distance represents time and vertical distance represents the quantity whose variation is in question. Since the variation is periodic, 
the curve will consist of repetitions of one and the same form, in other words it will consist of a number of equal and similar waves, and the wave-length stands for the complete period of the variation.

The point on which $I$ wish to insist is, that if such a curve representing the superposition of two harmonics of the fundamental is in the first instance very accurately drawn, and is then inaccurately copied in such a way that all successive waves are treated alike, the inaccuracy is morally certain to introduce the fundamental.

Let $y$ denote any ordinate, and $\theta$ the time (or abscissa) expressed in such a unit that $2 \pi$ is the numerical value of the wave-length or period; then the amplitude of the fundamental is the square root of $\mathrm{A}^{2}+\mathrm{B}^{2}$, where

$$
\mathrm{A}=\frac{1}{\pi} \int_{0}^{2 \pi} y \cos \theta d \theta, \quad \mathrm{B}=\frac{1}{\pi} \int_{0}^{2 \pi} y \sin \theta d \theta .
$$

In the original curve both $\mathrm{A}$ and $\mathrm{B}$ vanish. Let $y^{\prime}$ be the altered value of $y$ in the new and inaccurate curve, and let $z$ denote $y^{\prime}-y$; then we have, between the above limits,

$$
\int y^{\prime} \cos \theta d \theta=\int y \cos \theta d \theta+\int z \cos \theta d \theta=\int z \cos \theta d \theta
$$

since $\int y \cos \theta d \theta$ is zero.

But $z$ may be regarded as a random magnitude, hence it is infinitely improbable that its different values exactly fulfil the condition $\int z \cos \theta d \theta=0$. Therefore the new $A$ is finite; and similar reasoning shows that the new $B$ is finite.

If all the ordinates were changed in one uniform ratio, $\mathbf{A}$ and $B$ would remain zero, and no new constituent would be introduced; but any other change, unless specially planned to avoid introducing $A$ and $B$, is practically certain to give $\mathrm{A}^{2}+\mathrm{B}^{2}$ a finite value.

5. I maintain that such a change is effected in the form of sonorous waves during their transmission from the extermal air to the sensory fibres by which we distinguish pitch. The waves are transmitted first from the air to the drumskin, then through two successive levers, the hammer and anvil, to the head of the stirrup, while the foot of the stirrup sits upon the membrane of the oval window, and passes on the vibrations 
through the membrane to the liquid on the other side in which the sensory fibres are immersed. The levers turn upon ligamentous fulcra, and have rabbing contact with each other. The wave-form cannot run the gauntlet of all these transmissions without being to some extent knocked out of shape. It is much as if a very accurately drawn curve, representing the original wave-form, were copied and recopied, five times in succession, by five different pantagraphs not very firm in their connexions. The final copy so obtained would be sure to exhibit sensible departures from the original.

6. It appears likely that the chief seat of the disturbing actions in the ear is the junction of the hammer and anvil. "When the drumskin with the hanmer is driven outwards, the anvil is not obliged to follow it. The interlocking teeth of the surfaces of the joint then separate, and the surfuces glide over each other with very little f'iction "*. Such action is likely to introduce derangement, increasing generally with the excursions of the drumskin, but not expressible as a definite function of the ordinates of the wave-curve. For a given pressure on the drumskin, the pressure communicated to the liquid in the cochlea will vary according to the relative position and relative motion of the two portions of this joint.

7. The principal resultant tone due to these actions is likely to be that which corresponds to the complete period of the actions, in other words the highest common fundamental of the two primaries, or what old writers called the "grave harmonic." This will not be the same as the "first differencetone" unless the ratio of the two primaries is of the form $m: m+1$; and I have satisfied myself, both by my own trials and by a study of Kœnig's experimental results, that when the difference-tone and the common fundamental are not identical, the common fundamental is usually the predominant, and often the only audible resultant tone. (See Appendix.)

8. The cummon fundamental is, however, not the only resultant tone that can be thus accounted for. Similar reasoning to that employed in reference to $A$ and $B$ suffices to explain the introduction of any or all of the harmonics of

* Fllis's ' Helmholtz' p. 133, 2nd edition. 
the fundamental ; but it is to be expected, from the analogy of ordinary experience in harmonic analysis, that the successive constituents will usually be smaller and smaller as we advance in the series. The octave is likely to be the largest of them ; and $K œ n i g$ found, in several experiments with primaries in the ratio of $3: 5$, that both the fundamental 1 and its octave 2 were distinctly heard as resultant tones.

9. The following investigation bears on the relation between beats and resultant tones. The expression

$$
a \cos m \theta+b \cos n \theta
$$

cun be reduced to the form

$$
A \cos \left(\frac{m+n}{2} e-\epsilon\right)
$$

where $\mathrm{A}$ and $\epsilon$ are given by

$$
\begin{aligned}
\mathrm{A}^{2} & =a^{2}+b^{2}+2 a b \cos (n-m) \theta, \\
\tan \epsilon & =\frac{a-b}{a+b} \tan \frac{n-m}{2} \theta,
\end{aligned}
$$

and the beating together of two tones not differing much in pitch is explained by the fact, definitely expressed in these formulæ, that the whole effect may be regarded as a succession of waves with gradually varying amplitude. The frequency of the beats is the frequency of the maxima of $A^{2}$, and is the difference of $m$ and $n$.

We have ascribed resultant tones to alterations made in the wave-form by the action of the ear, such alterations being in general largest at those points at which the excursions of the drumskin are largest. These excursions are measured by $\pm A$, and the above investigation shows that their maxima have a frequency corresponding to the difference-tone. This is true whether $m$ and $n$ are commensurable or incommensurable. If they are commensurable, their greatest common measure will be the frequency of the complete cycle of change. This cycle will not be conspicuous in the curve if the ratio of $n-m$ to $\frac{1}{2}(n+m)$ is very small, but will assert itself more and more as this ratio increases; and these remarks will apply to the comparison of the fundamental with the first differencetone.

VOL. XIV, 
10. If the ear is able so to alter the form of waves impinging upon it as to generate resultant tones, it is natural to seek for some instance of a similar action in external bodies. A violin is very susceptible, like the ear, to vibrations of all frequencies between wide limits, and the sound-post serves, like the ossicles of the ear, to transmit vibrations from one portion to another. It is easy to produce resultant tones by bowing two strings of a violin together. For example, in the ordinary process of tuning, when the fourth and third strings with frequencies as $2: 3$ are combined, the resultant tone 1 is very observable if attention be directed towards it. But more striking effects are obtained when the resultant is at a larger interval from the primaries. The major sixth $3: 5$, the major second $8: 9$, and the minor seventh $5: 9$, are suitable intervals for calling out the fundamental 1 , the strings em. ployed being either the first and second or the second and third. The deep resultant tone thus obtained can not only be heard by the ear but felt as a tremor by the hand which holds the instrument. This is clear evidence of its objective existence, and I have succeeded in confirming the fact by means of a Helmholtz resonance-globe, the largest of the ordinary set, responding to $\mathrm{C}$ of 128 vibrations. When held with the edge of its mouth resting against the side of the violin, it responds to the combination $\mathrm{C}$ of 256 and $\mathrm{G}$ of 384 on the 4th and 3rd strings, or to the combination $\mathrm{C}$ of 512 and $E$ of 640 on the 2 nd and 1st ; or, still better, to the 3rd and 2 nd open strings each flattened one note, so as to be $\mathrm{C}$ of 256 and $G$ of 384 . Here, then, we have distinct evidence that the violin possesses the power which $I$ have ascribed to the ear-the power of manufacturing the fundamental when the two primaries are supplied.

11. Sir John Herschel, in his treatise on Sound (Enc. Met. arts. 238, 239), mentions the fact that the common fundamental can be called out by sounding two or more of its harmonics on very accurately tuned strings or pipes, and says that the effect cannot be obtained from a pianoforte tuned in the ordinary way, because the intervals are tempered. I find, however, on trying the experiment with an upright Broadwood of date about 1860 , that $\mathrm{C}$ of 64 is easily called out by simultaneously striking eight or ten of its harmonics; 
and the effect is greatly enhanced if the key of $\mathrm{C} 64$ is held down. In the latter case its note continues to be heard for a long time after the keys which were struck are released. From these experiments it appears probable that the soundingboard of a piano possesses the same property which we have proved to exist in the violin.

12. I now come to the explanation of the experiments of Professor Rücker and Mr. Edser (Proc. Phys. Soc. vol. xiii. p. 412 ; Phil. Mag. 1895, xxxix. p. 341). They were made with a Helmholtz siren, and in each instance the two primaries were produced in the same box, sometimes the upper and sometimes the lower box. The following explanation is a development of suggestions contained in Appendix xvi. of the Tonempindungen.

The rate of escape of air from the box containing the two rows of holes which are employed may as a first approximation be assumed to be jointly proportional, at each instant, to the aperture for escape and the differential pressure which produces the escape. Again, this differential pressure may be regarded as the algebraic sum of two terms, one of them constant, and representing its average value, while the other represents the difference from the average due to the varying amount of the aperture from instant to instant. As a first approximation, equal increments of aperture must be regarded as producing equal decrements of pressure, so that the variable term will be proportional (with reversed sign) to the excess of the aperture above its mean value. This excess (defect being counted negative) will be a periodic function of the time, and if the ratio of the two primaries in lowest terms be $m: n$, the frequency for the complete period will be represented on the same scale by 1 . In other words it will be the period of their common fundamental.

Let the aperture at time $t$ be expressed in a Fourier series, $\theta$ being put for $2 \pi t / \mathrm{T}$, where $\mathrm{T}$ is the complete period ; and let the variable part of the expression be denoted by $f(\theta)$, while $a_{0}$ denotes the mean aperture, so that the aperture at time $t$ is $a_{0}+f(\theta)$. We shall have $f(\theta)=A \sin \theta+\ldots+a_{1} \sin \left(m \theta+\epsilon_{1}\right)+\ldots+b_{1} \sin \left(n \theta+\epsilon_{2}\right)+$. The largest amplitudes will be $a_{1}$ and $b_{1}$ corresponding to the 
two primaries ; but A, which corresponds to the fundamental, is likely to be sensible.

The pressure at time $t$ is proportional to

$$
C-f(\theta)
$$

$\mathrm{C}$ being a constant; and the aperture is

$$
a_{0}+f(\theta) \text {. }
$$

Hence the rate of escape is proportional to

$$
\mathrm{C} a_{0}+\left(\mathrm{C}-a_{0}\right) f(\theta)-\{f(\theta)\}^{2}
$$

$a_{0}$ is comparable with the maxinum value of $f(\theta)$, and $C$ is much greater; hence $a_{0}$ may be neglected in comparison with C.

Developing $\left\{(f(\theta)\}^{2}\right.$, we shall obtain a term

$$
\begin{gathered}
2 a_{1} b_{1} \sin \left(m \theta+\epsilon_{1}\right) \sin \left(n \theta+\epsilon_{2}\right) \\
=a_{1} b_{1}\left[\cos \left\{(n-m) \theta+\epsilon_{2}-\epsilon_{1}\right\}-\cos \left\{(n+m) \theta+\epsilon_{2}+\epsilon_{1}\right\}\right],
\end{gathered}
$$

representing a difference-tone and a summation-tone. From $\left(\mathrm{C}-a_{0}\right), f(\theta)$ we have the common fundamental

$$
\left(\mathrm{C}-a_{0}\right) \mathrm{A} \sin \theta \text {, or } \mathrm{CA} \sin \theta \text {, }
$$

and the two primaries

$$
\mathrm{C} a_{1} \sin \left(m \theta+\epsilon_{1}\right), \quad C b_{1} \sin \left(n \theta+\epsilon_{2}\right) .
$$

Suppose for simplicity that $a_{1}=b_{1}$, then, taking the amplitude of each of the primaries as 1 , the amplitude of the common fundamental will be $A / a_{1}$, and the amplitudes of the summation-tone and difference-tone will each be $a_{1} / \mathrm{C}$.

When $n-m=1$, the difference-tone coincides with the fundamental, and their joint amplitude may be taken as the square root of the sum of the squares of $a_{1} / \mathrm{C}$ and $\mathrm{A} / a_{1}$.

13. Professor Rücker and Mr. Edser in experiments i. and ii. obtained the difference-tone 64 from five distinct combinations of primaries,

$256 \& 320,192 \& 256,320 \& 384,51 \cdot 2 \& 115 \cdot 2,96 \& 160$, their ratios being

$$
4: 5, \quad 3: 4, \quad 5: 6, \quad 4: 9, \quad 3: 5 \text {. }
$$

The second combination appears to have given a stronger effect than either the first or the third; whence it would appear that low frequencies are favourable to strong effects. 
Nevertheless the fourth combination is mentioned as giving a rather feebler effect than any one of the first three. This confirms our conclusion that the difference-tone is weaker when it is distinct from the common fundamental than when it coincides with it.

Experiment iii. was directed to testing for the presence of the resultant 64 when the primaries were 256 and 576, which are as $4: 9$. Their common fundamental is 64 , and it could not be detected. This may have been because the pitch 576 was too high to give a good effect. Or the failure may be an indication that $\mathrm{A} / a_{1}$ is decidedly smaller than $a_{1} / \mathrm{C}$. It would be interesting to repeat the experiment, employing 192 and 320 as the primaries.

14. Near the end of chapter vii. of the Tonempfindungen Helmholtz makes prominent mention of the slipping of the hammer on the anvil as an important cause of resultant tones, and appears to regard it as exemplifying his mathematical formula for the restoring force as a function of the displacement. But it is clear that if the hammer, which holds the drumskin, is liable to shift in its supports, the restoring force cannot be a mere function of the displacement, but must also depend on the relative position and relative velocity of the hammer and anvil at the moment considered. I accept all the consequences which Helmholtz deduces in the passage in question from this slipping, including its application to explain first difference-tones ; but I regard these consequences as lying outside the range of his general mathematical formulæe as given in Appendix xii.

15. To sum up my objections to the received mathematical theory of resultant tones :-

First. It assumes that the reaction of the drumskin against the air is a definite function of the displacement of the drumskin from a certain fixed position, whereas this reaction depends also on the position and motion of the further end of the bammer at the time.

Secondly. Even if the vibrations of the drumskin were in accordance with the received formulæ, there is plenty of scope for the introduction of additional constituents on the road from the drumskin to the liquid in the cochlea. The auditory ossicles, with their ligamentous supports and attachments, 
probably serve to protect the oval window of the cochlea against shocks and jars, and to smooth down asperities in the wave-form, thus mitigating the harshness of sounds and rendering them more musical. The changes thus introduced are very unlikely to fulfil the special conditions required for the vanishing of the common fundamental.

Thirdly. The received theory makes the common fundamental, when not coincident with the first difference-tone, depend on a term involving the cube or some higher power of the displacement. When the primaries are as $3: 5$, the fundamental 1 comes in as $2 m-n$, and depends on the cube of the displacement. When they are as $4: 11$, the tone 1 , which Konig found to be the loudest resultant, is $3 m-n$, and depends on the fourth power. When they are in the ratio $4: 15$, as in Kœnig's experiment with the simple tones $u t_{5}$ and $s i_{6}$, the common fundamental $u t_{3}$, which was the only resultant tone heard, is $4 m-n$, and depends on the fifth power of the displacement; the first difference-tone, which depends on the second power and should in theory be the loudest, being inaudible. This is surely a reductio ad absurdum of the received theory.

I do not wish to be understood as denying that the theory has any basis of truth. My contention is that the actions to which it is truly applicable play only a subordinate part in the production of resultant tones.

\section{APPENDIX.}

Examples selected from Kœnig's Expériences d'Acoustique, pp. 103 and 104, illustrating the production of the common fundamental. The "single vibrations" of the original are here reduced to double or complete vibrations :-

$u t_{5}$ and $s i_{5}$, which are as $8: 15$, gave only $u t_{2}$.

$u t_{5}$ and 2816 , which are as $4: 11$, gave $u t_{3}$ corresponding to 1 louder than any other tone.

$u t_{5}$ and $s i_{6}$, which are as $4: 15$, gave no audible tone but $u t_{3}$. $u t_{5}$ and 3968 , which are as $8: 31$, gave no audible tone but $u t_{2}$ 
$u t_{6}$ and 3584 , which are as $4: 7$, gave $u t_{4}$ more distinct than the difference-tone $s_{5}$.

$u t_{6}$ and $s i_{6}$, which are as $8: 15$, gave $u t_{3}$ distinct, the difference-tone 7 being inaudible.

$u t_{6}$ and 3968 , which are $16: 31$, gave $u t_{2}$ only.

$u t_{6}$ and 4032 , which are $32: 63$, gave $u t_{1}$ only.

\section{Discussion.}

Dr. C. V. Burton, after explaining several portions of Prof. Everett's paper, said that he (Dr. Burton) considered that the author's view in many ways seemed to fit in with the observed facts better than the accepted theory, but still did not appear itself quite free from objection. Prof. Everett supposes that the first term in a Fourier series is always the most important, and although in most cases which occur in practice this may be so, it hardly seems legitimate to take this as a characteristic of a Fourier series.

\section{The Magnetic Field of any Cylindrical Coil.}

By W. H. EveretT, B.A.*

\section{[Abstract.]}

Applying Ampere's formula for the magnetic force at any point due to an element of current, the force perpendicular to any plane circuit, carrying a current $i$, is found to be, at any point $P$,

$$
\mathrm{Z}^{\prime}=i \int \frac{r^{2} d \theta}{\left(r^{2}+h^{2}\right)^{\frac{3}{2}}} ;
$$

$h$ being the distance of $\mathrm{P}$ from the plane of the circuit, and $r, \theta$ the polar co-ordinates of any point of the circuit referred to the projection of $\mathbf{P}$ as origin.

The longitudinal force at any point due to a current in a cylindrical coil, or solenoid, is given by a second integration. It is the sum or difference of two terms, each of the form

$$
\mathrm{Z}=\operatorname{inh} \int \frac{d \theta}{\sqrt{r^{2}+h^{2}}}
$$

* Read November 8, 1895. 